\title{
Representation of convective plumes by vertical adjustment
}

\author{
Barry A. Klinger \\ Oceanographic Center, Nova Southeastern University, Dania, Florida
}

John Marshall

Center for Meteorology and Physical Oceanography, Department of Earth, Atmospheric, and Planetary Sciences, Massachusetts Institute of Technology, Cambridge

\section{Uwe Send}

Institut fuer Meereskunde, Kiel, Germany

\begin{abstract}
Open-ocean deep-water formation involves the interplay of two dynamical processes; plumes ( $\leq 1 \mathrm{~km}$ wide), driven by "upright" convection, and geostrophic eddies ( $\geq 5 \mathrm{~km}$ wide), driven by baroclinic instability. Numerical "twin" experiments are used to address two questions about the plumes: Can they be represented by a simple mixing process in large-scale models? If so, is it important that the mixing occurs over a finite time $t_{\text {mix }}$, or would instantaneous mixing produce the same effect on large-scale properties? In numerical simulations which resolve the geostrophic eddies, we represent the plumes with a "slow" convective adjustment algorithm which is broadly equivalent to an enhanced vertical diffusivity of density in statically unstable regions. The diffusivity $\kappa$ depends on $t_{\text {mix }}$, the mixing timescale. The fidelity of the plume parameterization is then evaluated by comparison with plume-resolving simulations of open-ocean deep convection. Integral properties of the plumes, such as the temperature census of the convected water and the strength of the rim current that encircles the convecting region, are all accurately reproduced by the slow adjustment scheme. The importance of choosing an appropriate finite value for $t_{\mathrm{mix}}$ is explored by setting $t_{\text {mix }}=12$ hours in some experiments, in accordance with scaling considerations, and $t_{\text {mix }}=0$ in others, corresponding to instantaneous adjustment, the conventional assumption. In the case of convection into a moderately or strongly stratified ocean the behavior does not significantly depend on $t_{\text {mix }}$. However, in neutral conditions the slow adjustment does improve the parametric representation. Our experiments confirm the picture of plumes homogenizing the water column over a time $t_{\text {mix }}$.
\end{abstract}

\section{Introduction}

Open-ocean convection is a significant source of deep and intermediate water masses and displays distinct structures on a wide spectrum of length scales. Deep water is actually formed in convection regions known as "chimneys" which have a scale of the order of $10-100 \mathrm{~km}$. Frequently, baroclinic instability breaks these chimneys into smaller geostrophic eddies, which can be less than $10 \mathrm{~km}$ wide, reflecting the small Rossby radius of the weakly stratified regions in which convection occurs. The convective elements themselves, the plumes, are less than a kilometer wide [Schott and Leaman, 1991; Schott et al., 1994] and exchange newly dense surface water with water from below [Jones and Marshall, 1993; Klinger and Marshall, 1995; Maxworthy and Narimousa, 1994; Marshall et al., 1994]. It is necessary to parameterize both the baroclinic instability and the convective plumes for use in large-scale, coarsely resolved numerical models used in climate research; here we test simple ideas about parameterizing the plumes.

Send and Marshall [1995] argue that the dominant effect of convective plumes is to vertically mix properties such as tem-

Copyright 1996 by the American Geophysical Union.

Paper number 96JC00861.

0148-0227/96/96JC-00861\$09.00 perature, salinity, and density. They characterize mixing by a timescale $t_{\text {mix }}$ which determines how long it takes before water properties at the bottom of a mixed layer are significantly changed by buoyancy forcing at the top. This timescale is the transit time of a particle as it descends, in a convective plume, from the sea surface to the bottom of the mixed layer. For convective plumes characterized by vertical speed $w_{\text {plume }}$ it takes a time of order

$$
t_{\text {mix }} \sim h / w_{\text {plume }}
$$

to bring dense surface fluid to the bottom. Here $h$ is the depth of the convecting layer and $w_{\text {plume }}$ is a vertical velocity scale for the plumes, observed to reach $3-10 \mathrm{~cm} / \mathrm{s}$ in field experiments [Schott and Leaman, 1991; Schott et al., 1994]. For deep ocean convection, $t_{\text {mix }}$ can be 12 hours or longer, perhaps long enough to be important for larger-scale processes such as geostrophic adjustment and baroclinic instability of the chimney.

A simple parameterization of convection can be used to represent this finite $t_{\text {mix }}$. For example, one could employ an appropriately chosen vertical diffusivity of temperature and salinity in statically unstable regions. Indeed, enhanced diffusion, with a somewhat arbitrarily chosen value of vertical diffusivity, is often used to parameterize convection [see Cox, 1984; Marotzke, 1991]. However, it is desirable to deduce the 
magnitude of the diffusivity $\kappa$ from physical knowledge of $t_{\text {mix }}$. Since a diffusive system takes a time of order $h^{2} / \kappa$ to send a signal a distance $h$, the diffusivity ought to be [see Send and Marshall, 1995]

$$
\kappa \sim h^{2} / t_{\mathrm{mix}} \sim h w_{\text {plume }}
$$

For $w_{\text {plume }}=0.05 \mathrm{~m} / \mathrm{s}$ and $h=1000 \mathrm{~m}, \kappa \sim 50 \mathrm{~m}^{2} / \mathrm{s}$. The actual value of $\kappa$ appropriate to our numerical experiments described below is about $10 \mathrm{~m}^{2} / \mathrm{s}$. Both values are significantly larger than the value of $1 \mathrm{~m}^{2} / \mathrm{s}$ used by Marotzke [1991].

In the limit in which $\kappa \rightarrow \infty$, the mixing timescale goes to zero so that the convection region is vertically homogeneous at all times. This corresponds to the traditional convective adjustment scheme which homogenizes any statically unstable segment of the water column at each time step [Bryan, 1969; Marotzke, 1991; Yin and Sarachik, 1994]. This instantaneousmixing convective-adjustment (IMCA) scheme is a cruder representation of the process than enhanced diffusivity because it does not take into account the finite time it takes plumes to exchange properties.

In this paper we answer two questions concerning the mixing properties of plumes. If convective plumes are represented by diffusive vertical mixing, to what extent are geostrophic scales faithfully reproduced? How important is it to incorporate a realistic estimate of $t_{\mathrm{mix}}$ when representing plumes parametrically?

In section 2 we determine the circumstances under which a finite convective timescale might be expected to have a significant effect on the properties of the convection region. In Appendix A we introduce a slow (finite $t_{\text {mix }}$ ) convectiveadjustment scheme which we show to be physically equivalent to an enhanced vertical diffusivity parameterization. Unlike an enhanced diffusivity, the scheme does not require implicit time stepping for numerical stability. Section 3 compares numerical experiments in which convective plumes are present with experiments in which convection is parameterized with the slow adjustment scheme. The importance of $t_{\mathrm{mxx}}$ is studied by comparing finite $t_{\mathrm{mix}}=12$-hour runs with $t_{\mathrm{mix}}=0$ runs.

In the experiments, cooling is restricted to a circular disk at the center of a doubly periodic domain (as given by Jones and Marshall [1993]), in order to mimic some of the consequences of localized preconditioning and localized forcing. In particular, the periphery of the cooling region develops a "rim current" which is baroclinically unstable and grows meanders which pinch off to form eddies. These eddies have a critical influence on the ultimate properties of the newly formed deep water. The richness of the behavior of this system, with its rim current properties, baroclinic instability patterns, and water mass evolution, provides a critical test of the appropriateness of the parameterization scheme. It allows us to measure the scheme's influence on key chimney properties which in turn control the ultimate water mass properties of the newly formed dense water.

Two physical contexts are used to test the parameterizations. In the first the ocean has an initially uniform density, so that the instantaneous mixing scheme immediately transmits the density signal to the bottom of the water column. In the second the ocean has an initially uniform density gradient, so that the density signal must "eat away" at the stratification before it can reach the bottom.

We neglect nonlinearities in the equation of state of seawater, such as the thermobaric effect, which may have important effects in the coldest regions in which deep convection occurs, notably the Greenland and Weddell Seas [Garwood et al., 1994; Paluszkiewicz, 1994; D. Denbo and E. Skyllingstad, An ocean large eddy model with application to deep convection in the Greenland Sea, submitted manuscript, 1994]. The thermobaric effect, however, is negligible in the Mediterranean and probably small in the Labrador Sea. For example, evaluation of the equation of state in the roughly $3^{\circ} \mathrm{C}$ water of the top kilometer of the Labrador Sea shows that $\partial \rho / \partial \theta$ can be approximated to within $12 \%$ by a constant value; in colder water, $\partial \rho / \partial \theta$ displays greater sensitivity to pressure. Numerical experiments by Sander et al. [1995], in which thermobaric effects are included in seawater of about $0^{\circ} \mathrm{C}$, show a strong effect on the plume properties but a weaker effect on the average temperature profile. For a linear equation of state the density equation behaves in the same manner whether fresh water or heat fluxes are imposed. For convenience, then, we treat density as a function of temperature only.

\section{Timescales for Oceanic Convection}

Laboratory experiments [Maxworthy and Narimousa, 1994; Fernando et al., 1991; Boubnov and Golitsyn, 1986, 1990] and numerical simulations [Jones and Marshall, 1993; Klinger and Marshall, 1995] have investigated the characteristics of convective plumes in an initially homogeneous fluid of depth $H$, subject to surface buoyancy forcing $B$, in a system with a Coriolis parameter $f$, viscosity $v$, and diffusivity of density (i.e., of either salinity or temperature depending on the system) $\kappa$. When the flux Rayleigh number, $R a_{f}=B H^{4} / \kappa^{2} \nu$, is sufficiently large ( $R a_{f} \approx 10^{27}$ in the ocean), the system becomes insensitive to $R a_{f}$ and is governed by the natural Rossby number, $R o^{*}=\left(B / f^{3} H^{2}\right)^{1 / 2}$ (for a review of the physical importance of $R o^{*}$, see Marshall et al. [1994]). The scales for the convective vertical velocity are

$$
\begin{array}{cl}
w_{\text {plume }} \sim(B H)^{1 / 3} \equiv U_{\text {non }} & \left(R o^{*} \geq 1\right) \\
w_{\text {plume }} \sim(B / f)^{1 / 2} \equiv U_{\text {rot }}=U_{\text {non }} R o^{* 1 / 3} & \left(R o^{*} \ll 1\right) .
\end{array}
$$

For typical depth scales of order $1 \mathrm{~km}, B$ of order $10^{-7} \mathrm{~m}^{2} / \mathrm{s}^{3}$ (equivalent to heat flux of the order of $500 \mathrm{~W} / \mathrm{m}^{2}$ ), and $f \approx$ $10^{-4} \mathrm{~s}^{-1}, R o^{*} \approx 0.1$. In a parameteric representation of a mixed layer the appropriate scaling for $w_{\text {plume }}$ can be selected based on a calculation of $R o^{*}$ from $f, B$, and either $H$, the total depth of the ocean (if the density is initially homogeneous), or $h$, the thickness of the mixed layer. As the depth of the mixed layer increases over the course of the winter, the natural Rossby number decreases so that $w_{\text {plume }}$ progresses from the nonrotational regime to the rotational regime (if $h$ becomes sufficiently deep). As the convective layer deepens, $w_{\text {plume }}$ increases from 0 to (at most) $U_{\text {rot }}$.

Given $w_{\text {plume }}$, and hence (by (1)) $t_{\mathrm{mix}}$, we can estimate how long it takes for the bottom of a homogeneous layer to be affected by a buoyancy flux at the surface. Numerical results (section 3.1) indicate that a proportionality constant of about 1.3 is needed to make the scale relation (1) an equality, so that for $H=2 \mathrm{~km}, B=4 \times 10^{-7} \mathrm{~m}^{2} / \mathrm{s}^{3}$, and $f=10^{-4} / \mathrm{s}$ (as used below), $t_{\mathrm{mix}} \approx 12$ hours. This is long enough to potentially influence the evolution of the rim current and the eddies it generates, so that $t_{\text {mix }}$ may be important in a homogeneous or weakly stratified ocean.

If the ocean is stratified, however, the transport of dense fluid from the surface is delayed not only by the time it takes convective plumes to carry water down but also by the time 
taken to erode the preexisting thermocline. If this latter timescale $t_{0}$ is longer than $t_{\text {mix }}$, then $t_{\text {mix }}$ will not be of importance in the case of a stratified ocean subjected to constant surface forcing. We calculate $t_{0}$ assuming instantaneous mixing in which the mixed layer density is equal to the density just below the mixed layer. Given initial stratification specified by the uniform buoyancy frequency $N$, and assuming that entrainment fluxes through the base of the mixed layer can be neglected, $t_{0}$ can be obtained from

$$
h=\sqrt{2 B t} / N .
$$

Instantaneous mixing incorrectly predicts the time it takes convection to reach a given depth if $t_{0} \ll t_{\text {mix }}$, where $t_{\text {mix }}$ is given by (1) and (3). Thus an instantaneous mixing assumption will be inadequate if

$$
\begin{array}{cc}
(N / f)<\sqrt{2} R o^{* 2 / 3} & \left(R o^{*} \geq 1\right) \\
N<\sqrt{2}\left(B / h^{2}\right)^{2 / 3} & \left(R o^{*} \geq 1\right)
\end{array}
$$

or

$$
\begin{array}{lc}
(N / f)<\sqrt{2} R o^{* 1 / 2} \quad\left(R o^{*} \ll 1\right) \\
N<\sqrt{2}\left(B f / h^{2}\right)^{1 / 4} \quad\left(R o^{*} \ll 1\right) .
\end{array}
$$

Typically, for deep convection, $R o^{*}$ is at most $\mathrm{O}(1)$. The criteria (5) imply that the initial stratification must be very small for the finite $t_{\text {mix }}$ to affect the large-scale behavior of a convection region. $(N / f)=1$ implies a change in density of only $0.001 \mathrm{~kg} / \mathrm{m}^{3}$ for every $1 \mathrm{~km}$ of depth, which is near the limit of instrument resolution for oceanographic measurements. Thus we might expect that a finite $t_{\text {mix }}$ is only significant in the case of convection in an essentially unstratified ocean.

\section{Test of Slow Adjustment Scheme}

\subsection{Procedure}

The performance of the slow convective-adjustment algorithm (see Appendix A for details) was tested in simulations of convective chimneys in stratified and neutral oceans. Numerical experiments in which mixing was parameterized by the adjustment algorithm were compared with reference experiments in which the water column was mixed by resolved convection plumes. Table 1 summarizes the experiments, Table 2

Table 1. Summary of Numerical Experiments

\begin{tabular}{cccc}
\hline & Unparameterized & $\begin{array}{c}\boldsymbol{t}_{\text {mxx }}= \\
12 \text { hours }\end{array}$ & $\begin{array}{c}\boldsymbol{t}_{\text {mix }}= \\
\mathbf{0} \text { hours }\end{array}$ \\
\hline $\begin{array}{c}\text { Homogeneous, } \\
t_{C}=6 \text { days, }\end{array}$ & $\mathrm{H} 1$ & $\mathrm{HF} 1$ & $\mathrm{HI} 1$ \\
$\begin{array}{c}t_{R}=6 \text { days } \\
\text { Homogeneous, }\end{array}$ & $\mathrm{H} 2$ & $\mathrm{HF} 2$ & $\mathrm{HI2}$ \\
$t_{C}=2$ days, & & & \\
$t_{R}=4$ days & $\mathrm{S}$ & $\mathrm{SF}$ & $\mathrm{SI}$ \\
$\begin{array}{c}\text { Stratified, } \\
t_{C}=10 \text { days, }\end{array}$ & & & \\
$t_{R}=10$ days & & &
\end{tabular}

Numerical experiment titles. Experiments listed in the same row have same initial condition and forcing; experiments listed in the same column have the same parameterization of convective plumes. Here $t_{C}$ is duration of cooling and $t_{R}$ is duration of experiment. Abbreviations are $\mathrm{H}$, homogeneous initial condition; $\mathrm{S}$, stratified initial condition; $\mathrm{F}$, finite $t_{\mathrm{mix}} ; \mathrm{I}$, instantaneous $t_{\mathrm{mux}}$.
Table 2. Experimental Parameters

\begin{tabular}{lll}
\hline \multicolumn{1}{c}{ Definition } & Variable & \multicolumn{1}{c}{ Value } \\
\hline Surface heat flux & $Q$ & $875 \mathrm{~W} / \mathrm{m}^{2}$ \\
Surface buoyancy flux & $B$ & $4.62 \times 10^{-7} \mathrm{~m}^{2} / \mathrm{s}^{3}$ \\
Cooling disk diameter & $r$ & $16 \mathrm{~km}$ \\
Depth & $H$ & $2130 \mathrm{~m}$ \\
Coriolis parameter & $f$ & $10^{-4} / \mathrm{s}$ \\
Natural Rossby number & $R o^{*}$ & 0.32 \\
Horizontal grid spacing & $\Delta x$ & $250 \mathrm{~m}$ \\
Vertical grid spacing & $\Delta z$ & $112 \mathrm{~m}$ \\
Reference density & $\rho_{0}$ & $1000 \mathrm{~kg} / \mathrm{m}^{3}$ \\
Reference temperature & $\theta_{0}$ & $12^{\circ} \mathrm{C}$ \\
Expansion coefficient & $\alpha$ & $2.1 \times 10^{-4} /{ }^{\circ} \mathrm{C}$ \\
Background buoyancy frequency & $N$ & $4.79 \times 10^{-4} / \mathrm{s}$ \\
Vertical diffusivity & $\kappa_{\nu}$ & $0.2 \mathrm{~m}{ }^{2} / \mathrm{s}$ \\
Horizontal diffusivity & $\kappa_{H}$ & $5 \mathrm{~m}^{2} / \mathrm{s}$ \\
Horizontal flux Rayleigh number & $R a_{f}$ & $7.6 \times 10^{4}$ \\
\hline
\end{tabular}

displays all parameters, and Appendix B provides further details. Figure 1 shows the flow field in experiment H1 (homogeneous initial condition) after 1 day of cooling, when approximately kilometer-wide plumes reaching from surface to bottom can be seen in the temperature and velocity fields.

In parallel with the plume-resolving experiments, plumeparameterizing experiments were conducted with the adjustment scheme outlined in Appendix A. The adjustment scheme was employed with both finite $t_{\text {mix }}$ and (instantaneous) $t_{\text {mix }}=$ 0 . Each plume-parameterizing experiment was identical to the corresponding reference experiment except as noted. We define $t_{\mathrm{mix}}$ to be the time at which the temperature in the bottom level starts to display a significant rate of cooling. In experiments $\mathrm{H} 1$ and $\mathrm{H} 2, t_{\mathrm{mix}} \approx 12$ hours. For the values of $H$ and $w_{\text {plume }}$ given by Table 2 and (3), the value of $t_{\text {mix }}$ deduced by inspection of numerical simulation suggests that the numerical value of $t_{\text {mix }}$ should be about a factor of 1.3 greater than the timescale $H / w_{\text {plume }}$ stated in (1). According to (8), $t_{\text {mix }}=12$ hours is equivalent to an effective vertical diffusivity of $\kappa \approx 9$ $\mathrm{m}^{2} / \mathrm{s}$.

In experiment $S$ (initially stratified), $t_{\text {mix }}$ increased from zero as the statically unstable layer deepens. However, since the analysis of section 2 predicts that $t_{\mathrm{mix}}$ should not have a significant impact on the behavior, the slow adjustment scheme was simply implemented with $t_{\text {mix }}=12$ hours, in order to provide an upper bound on the influence (if any) of finite $t_{\text {mix }}$ in this case.

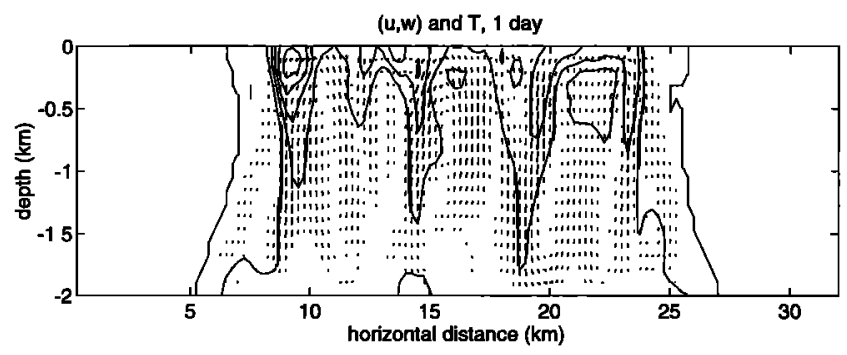

Figure 1. Velocity and temperature fields, experiment H1, in vertical section through center of cooling region, day 1 , showing statically unstable stratification and plumes. 

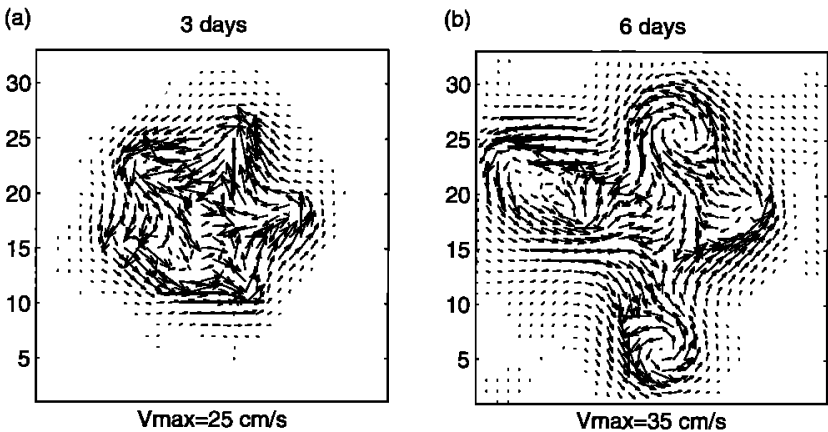

Figure 2. Velocity fields, experiment $\mathrm{H} 1$, depth of $112 \mathrm{~m}$, days (a) 3, and (b) 6. Vmax is largest speed in field.

\subsection{Homogeneous Experiment Results}

We first examine the runs in which cooling was applied for 6 days. In the plume-resolving experiment, H1, the following sequence of events occurred. As the density of the cylinder of water underneath the cooling disk increased, rim currents formed, flowing cyclonically around the cylinder near the surface and anticyclonically near the bottom. Baroclinic instability then formed meanders in these currents which grew until eddies formed (Figure 2). Rim currents and baroclinic instability also developed in experiment HF1 (convective adjustment scheme with $t_{\operatorname{mix}}=12$ hours; see Figure 3 ) and experiment HI1 $\left(t_{\text {mix }}=0\right.$; see Figure 4). The maximum horizontal speed measured near the surface every 24 hours in experiment HF1 was always within about $20 \%$ of the maximum speed in experiment H1. Maximum speeds in HI1 were similarly close to experiment $\mathrm{H} 1$ except for the first day, when the flow was somewhat slower than in experiment HF1. The meanders in experiment HF1 grew about as quickly as those in experiment $\mathrm{H} 1$, while those in HI1 grew at a somewhat slower rate.

The formation of "new" water is best charted by a water mass census showing the volume of water in each of several temperature ranges. Figure 5 displays such a census for the unstratified experiments; volume is measured by the number of grid points in the domain whose temperature falls within each $0.002^{\circ} \mathrm{C}$ temperature interval. Volume is normalized by that of the cylinder of water covered by the cooling disk. At the end of the first day the slow adjustment scheme reproduced the temperature range of the newly formed water observed in the unparameterized experiment (Figure 5a), though it did not display the distinct peak at $11.994^{\circ}$ seen in $\mathrm{H} 1$. In contrast, in experiment HI1 the instantaneous mixing caused the whole (a)

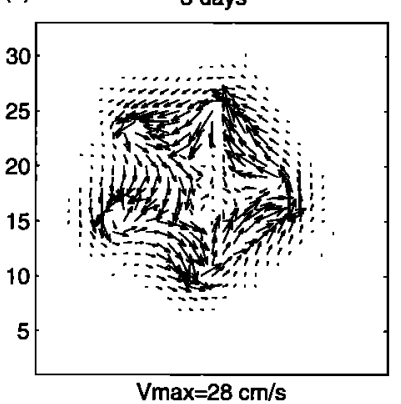

(b)

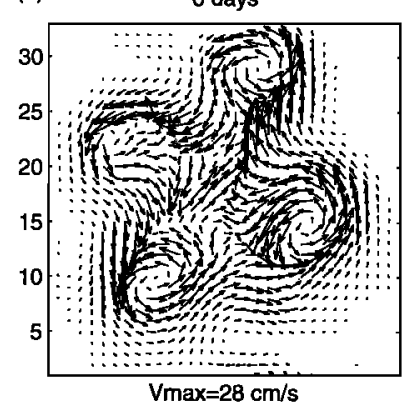

Figure 3. Velocity fields, experiment HF1, depth of $112 \mathrm{~m}$, days (a) 3 and (b) 6 .
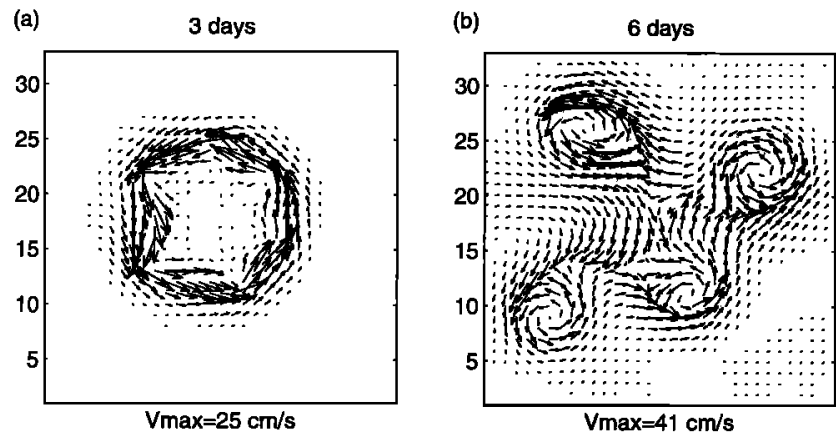

Figure 4. Velocity fields, experiment HI1, depth of $112 \mathrm{~m}$, days (a) 3 and (b) 6 .

water column to be at a single temperature at day 1 . Only a small volume of water around the edges attained an intermediate temperature as parcels moved into or out of the convection region. Experiment HI1's unrealistically sharp peak in the temperature census persisted into the third day; during the same period the temperature census in experiment HF1 grew closer to that of the plume-resolving experiment (Figure $5 b$ ).

After the third day, however, the characteristics of the temperature census were dominated by the effects of baroclinic instability. As eddies exchanged water between the cooling
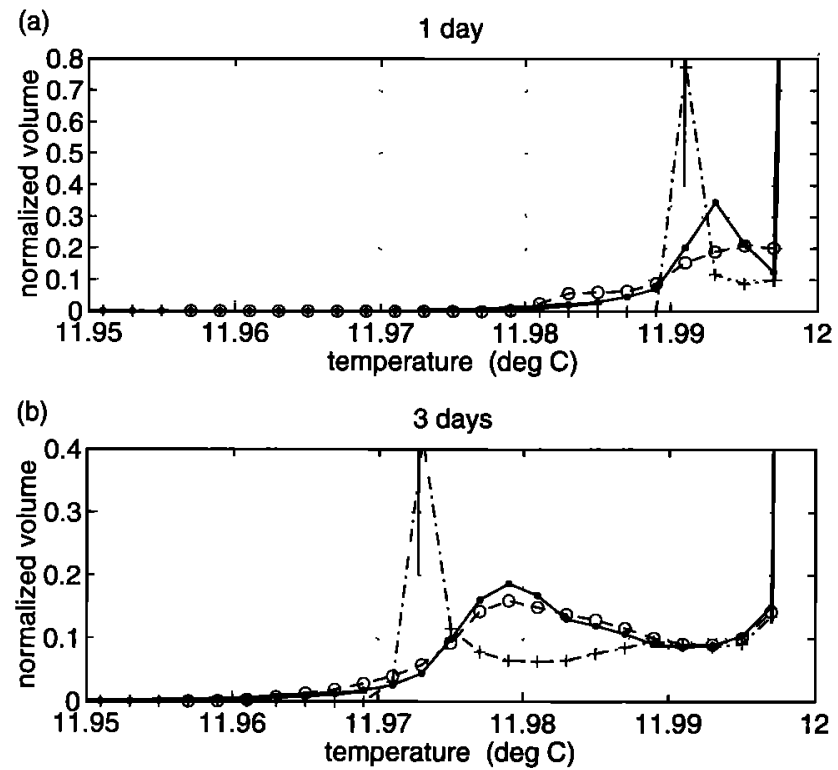

(c)

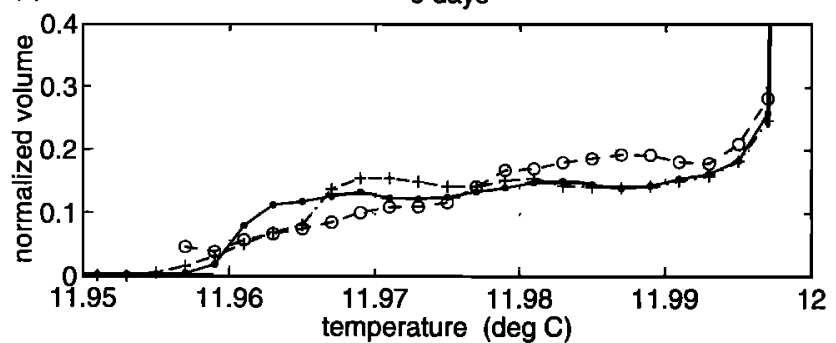

Figure 5. Temperature census, experiments H1 (solid line), HI1 (dashed-dotted line), and HF1 (dashed line) at days (a) 1, (b) 3, and (c) 6. Vertical line in Figures 5a and 5b marks the temperature modified water would have if it were isolated from surrounding water and instantaneously mixed. 


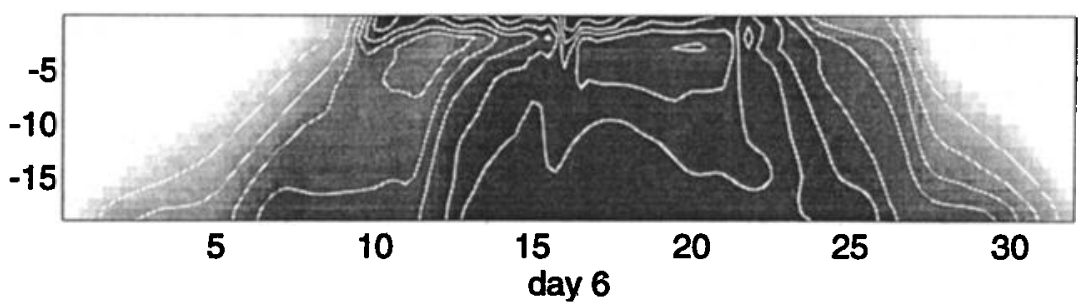

Figure 6. Vertical section of temperature through center of cooling region, experiment $\mathrm{H} 1$, day 6 . Temperature contours are 11.93 to $12^{\circ} \mathrm{C}$ with contour interval $0.005^{\circ} \mathrm{C}$.

region and the exterior, the surface heat flux cooled an increasing volume of water rather than further cooling a fixed volume. Thus the temperature of the peak in the histogram changed much less rapidly as time progressed. During this period, both the slow and instantaneous adjustment reproduced the temperature census fairly well (Figure 5c).

The details of the convection parameterization scheme were more important in the first half of the experiment than during the second half. Once meanders and eddies became prominent, they tended to restratify the domain by exporting dense fluid from the cooling region at the bottom and importing light fluid near the surface (Figure 6). As long as buoyancy is removed from the surface, there must be static instability in the top of the water column. However, after 3 days in experiment $\mathrm{H} 1$, static instability below the top few hundred meters of the water column was only present in about $20 \%$ of $A$, the cooling disk area. This was an even smaller fraction of the total area affected by cooling, which increased to several times $A$ as the geostrophic eddies spread out from the cooling disk. The sparsity of deep convection indicates that baroclinic instability dominated upright convection in most of the water column after a day or two.

The experiments in which cooling ceased after 2 days exhibited similar behavior to those which had continuous cooling. The cessation of cooling allowed somewhat more distinct eddies to emerge within the first 2 days. As in the case of continuous cooling, experiments with parameterized convection reproduced realistic current speeds. The temperature census (Figure 7) shows that in both $\mathrm{H} 2$ and $\mathrm{HF} 2$, the "cold tail" that was present during cooling (Figure 5b) disappeared 1 day after cooling ceased. Evidently both the plumes (in $\mathrm{H} 2$ ) and the adjustment scheme (in HF2 and HI2) efficiently homogenize the statically unstable fluid,

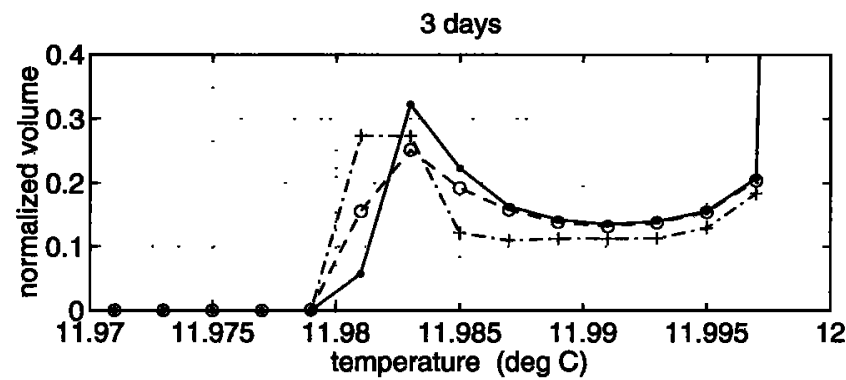

Figure 7. Temperature census, experiments $\mathrm{H} 2$ (solid line), HI2 (dashed-dotted line), and HF2 (dashed line) at day 3.

\subsection{Stratified Experiment Results}

Cooling an initially stratified fluid induced a large-scale flow which was similar to that of the unstratified experiments, but here the active layer grew downward from the surface until a quasi-steady state was reached. As argued in section 2 , one would not expect large-scale properties to be sensitive to details of the parameterization scheme for our test case of $N / f=$ 5. Figures 8 and 9 show that the flow fields were indeed strikingly similar in both the reference experiment $S$ and the slow adjustment scheme experiment SF. The instantaneous mixing experiment SI was also nearly identical, as was the temperature census (Figure 10).

\section{Conclusions}

We can now answer the two questions posed in the introduction. The first question asked whether the net effect of the convective plumes could be represented by a vertical adjustment process. We compared numerical experiments employing a modified convective adjustment scheme with numerical experiments in which plumes were explicitly resolved. Following the ideas of Send and Marshall [1995], the adjustment was designed to occur on a timescale $t_{\mathrm{mix}}$ dependent on the velocity scales of the plumes and the depth of the convective layer. Such a parameterization of convection is equivalent to employing a large vertical diffusion of buoyancy in statically unstable regions. For one set of parameters typical of deep convection, our analysis indicates that $\kappa \approx 10 \mathrm{~m}^{2} / \mathrm{s}$ is appropriate for vigorous plumes. The adjustment parameterization successfully reproduced the effects of such plumes on the chimney, its water mass properties, rim current speeds, and lateral exchange between the convective region and the exterior due to baroclinic instability. Thus a mixing model of convection is quite adequate to represent gross properties of the resolved plumes seen in our numerical experiments.

The second question concerned the importance of using a finite timescale for vertical mixing. Model experiments using the appropriate value of $t_{\text {mix }}$ (12 hours) were compared with others that employed instantaneous mixing. In unstratified conditions the use of a finite mixing timescale improved the temperature census in the early stages of convection, but instantaneous mixing was found to be perfectly adequate in the stratified experiments. Here the key timescale was determined by how long it took a mixed layer to penetrate the stratification rather than by $t_{\text {mix. }}$.

These results are rather encouraging because they support the simplifying ideas developed by Send and Marshall [1995] that convective plumes are mainly mixing agents that distribute the buoyancy deficit throughout the water column. They are 

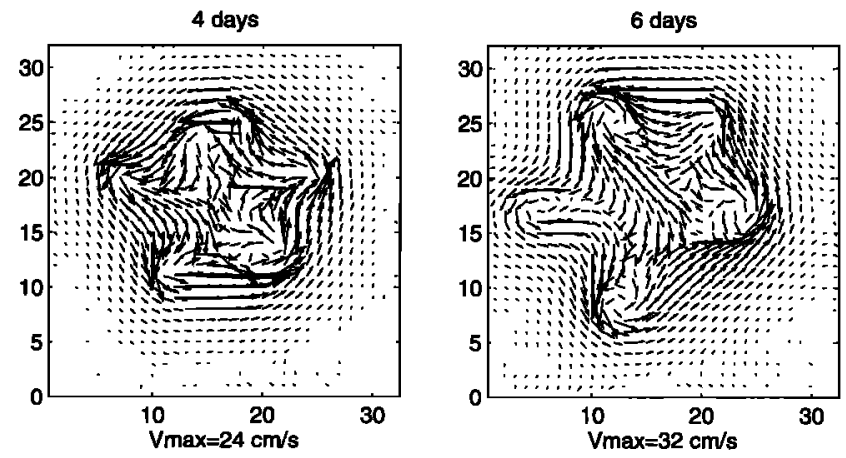

Figure 8. Velocity fields, experiment $\mathrm{S}$, depth of $112 \mathrm{~m}$, days 4 and 6.

also supported by field observations. A wealth of hydrographic data shows that deep convection rapidly and efficiently homogenizes property profiles to great depth [MEDOC Group, 1970; Leaman and Schott, 1991; Schott and Leaman, 1991]. The mixing does not necessarily create horizontally homogeneous conditions [Schott et al., 1994], due to spatially varying initial stratification, but the vertical-mixing view of convection is strongly supported by the success of one-dimensional mixedlayer simulations of observed convection [Mertens, 1994; Send et al., 1995].

The results suggest that simple mixing ought to be sufficient to represent the plumes in a convective chimney. A corollary is that if we are primarily interested in the gross properties of the chimney and its water mass formation, field work does not need to resolve the plume dynamics. Instead, it appears to be sufficient to observe the evolution of the stratification from the geostrophic scale (5 km and upward). The emphasis can then be on the horizontal processes (breakup and spreading) which are less well understood but of greater importance for the water masses and circulation. These conclusions may not apply to the coldest convection sites, such as the Greenland Sea, where thermobaric effects may give the properties of individual plumes greater significance. Thermobaric effects are not expected to be of first-order importance in such deep convection regions as the Labrador and western Mediterranean Seas.

Finally, we addressed the technical matter of parameterizing convection in numerical models. We showed that mixing schemes can adequately represent the action of plumes and that the timescale of adjustment does not appear to be of prime importance, except perhaps in the case of convection in
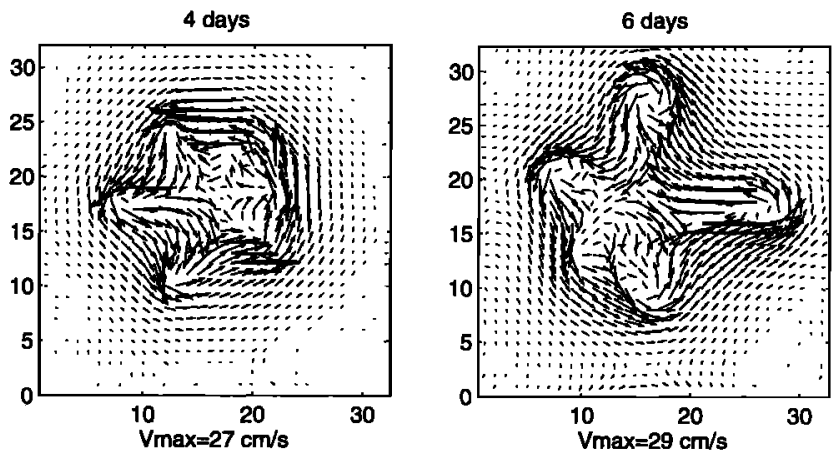

Figure 9. Velocity fields, experiment SF, depth of $112 \mathrm{~m}$, days 4 and 6.

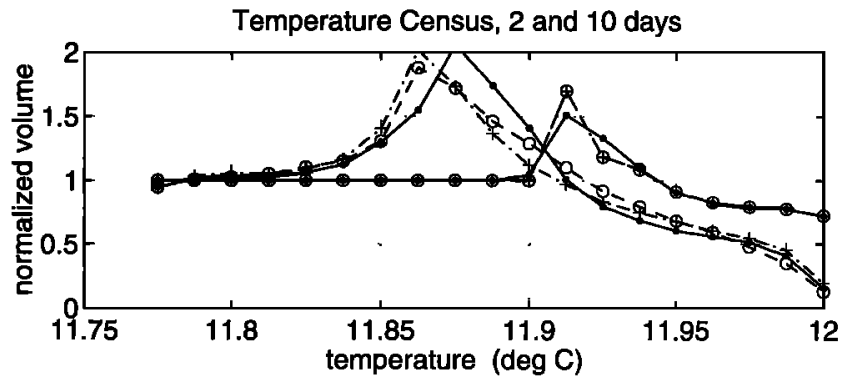

Figure 10. Temperature census, days 2 and 10, for experiments S (solid line), SI (dashed-dotted line), and SF (dashed line).

a neutral ocean. A complete parameterization of convection, however, must also incorporate lateral transfer by the geostrophic eddies that are central to controlling the volume and TS properties of convectively modified water.

\section{Appendix A: Slow Convective-Adjustment Scheme}

Our slow adjustment scheme is a modified instantaneousmixing convective-adjustment (IMCA) algorithm. The IMCA scheme instantaneously mixes density throughout a statically unstable region so that it is set to a uniform value. The slow convection scheme also vertically mixes density, but it does not completely homogenize the water column until the surface cooling ceases, and it takes a finite time $t_{\operatorname{mix}}$ to propagate surface density changes down the entire statically unstable region.

Suppose that the convective layer is represented by evenly spaced levels, a distance $\Delta z$ apart, as shown in Figure 11. At every horizontal position the algorithm searches for the deepest pair of adjacent levels in which the upper level is denser than the lower level. At such a statically unstable jump, density is mixed downward $\mathcal{N}_{\text {mix }}$ levels, where $\mathcal{N}_{\text {mix }}$ will be chosen to imply an appropriate $t_{\mathrm{mxx}}$. Suppose the upper level, labeled by index $i=I$, has density $\rho_{\mathrm{old}}=\rho_{0}+\Delta \rho$, and the lower level, $i=I+1$, has density $\rho_{\mathrm{old}}=\rho_{0}$ (the index increases from the top of the water column to the bottom). The algorithm determines $\mathcal{N}_{H}$, the number of contiguous levels above level $I+1$ which have the same density as level $I$. The density anomaly $\Delta \rho$ is then redistributed from these $\mathcal{N}_{H}$ levels so that

$$
\rho_{\text {new }}=\rho_{\text {old }}-\Delta \rho+\frac{\mathcal{N}_{H}}{\mathcal{N}_{H}+\mathcal{N}_{\text {mix }}} \Delta \rho \quad \text { levels }\left(I-\mathcal{N}_{H}+1\right) \text { to } I
$$

$$
\rho_{\text {new }}=\rho_{\text {old }}+\frac{\mathcal{N}_{H}}{\mathcal{N}_{H}+\mathcal{N}_{\text {mix }}} \Delta \rho \quad \text { levels }(I+1) \text { to }\left(I+\mathcal{N}_{\text {mix }}\right)
$$

(see Figure 11b). The algorithm then searches up the water column for the next statically unstable pair of levels.

Consider an initially homogeneous fluid cooled from above in which there is no other dynamics. When the slow adjustment scheme is used, steps of statically unstable density appear first at the top of the water column, where they are generated by the surface forcing. Over time they propagate downward (Figure 12). The whole water column remains statically unstable, a 

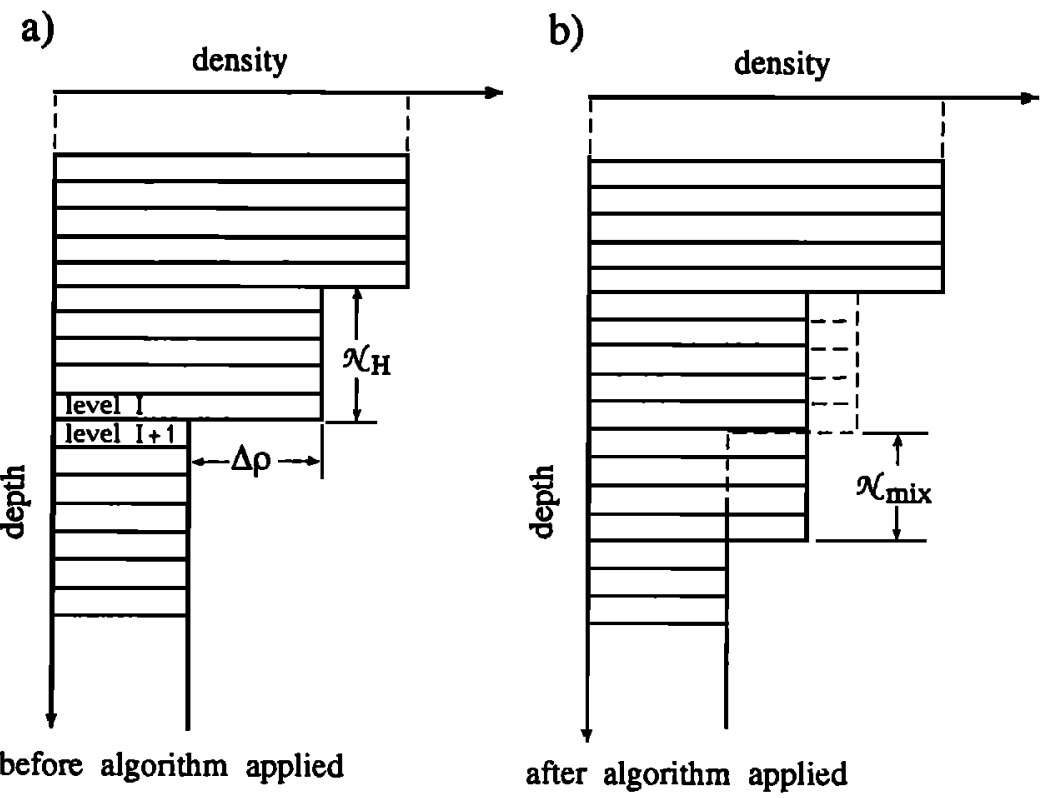

Figure 11. Illustration of redistribution of density in slow convective-adjustment scheme: (a) before algorithm was applied and (b) after algorithm was applied.

realistic feature since in any convecting fluid, it is the static instability which drives the convective mixing.

By considering the flux of density transferred from level to level, it can be shown mathematically that the effect of the slow adjustment algorithm is approximately equivalent to that of an enhanced vertical diffusion, with vertical diffusivity $\kappa$ given by

$$
\kappa=c \frac{\left(\Delta z \mathcal{N}_{\text {mix }}\right)^{2}}{\Delta t}
$$

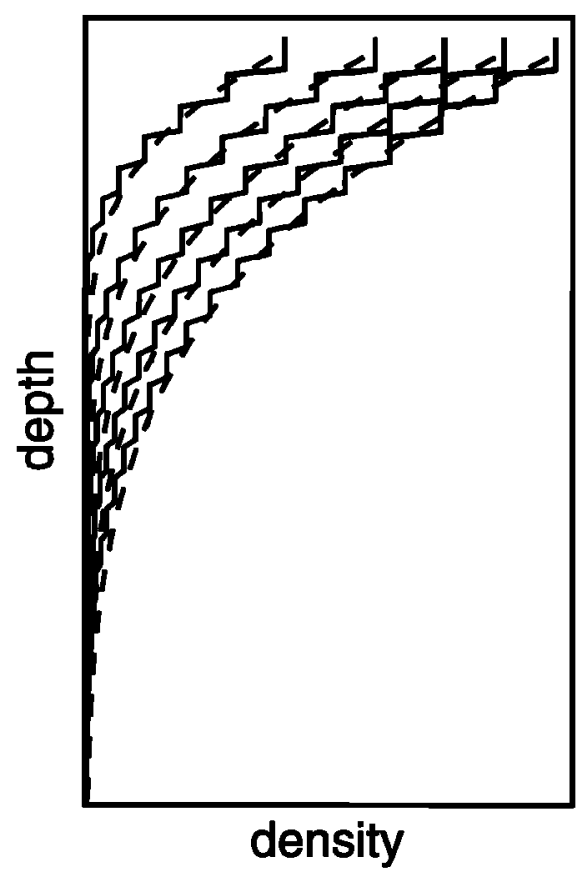

Figure 12. Comparison of slow convection-adjustment scheme (solid line) with enhanced diffusion (dashed line) in one-dimensional computation. Profile of density as function of depth for first few time steps. where $c$ is a nondimensional number. In one-dimensional simulations with density added at the surface the observed similarity between the behavior of the slow adjustment scheme and actual diffusion (Figure 12) confirms this equivalence. Defining $t_{\text {mix }}$ to be the time after initiation of cooling at which the bottom density $\rho_{\mathcal{N}}(t)$ begins to change, guidance from the simulations allows us to write (2) more exactly as

$$
\kappa \approx 0.1 h^{2} / t_{\mathrm{mix}}
$$

and we find that in $(7), c \approx 0.6$. When we actually implement the parameterization, we must determine $\mathcal{N}_{\text {mix }}$ as a function of $t_{\text {mix }}$. For $h=\mathcal{N} \Delta z,(7)$ and (8) and $c=0.6$ yield

$$
\mathcal{N}_{\mathrm{mix}}=0.4 \mathcal{N} \sqrt{\frac{\Delta t}{t_{\mathrm{mix}}}} .
$$

The slow adjustment scheme described here is not the only algorithm which acts like an enhanced vertical diffusion. The original convective-adjustment scheme of the Cox-Bryan model [Bryan, 1969], in which each pass of the algorithm mixes adjacent levels, also displays the same behavior as solutions to the diffusion equation. The more passes performed at each time step, the larger the effective $\kappa$ is and the more quickly the whole water column is mixed.

\section{Appendix B: Numerical Details and Parameters}

All numerical experiments employed the incompressible Navier Stokes model described by J. Marshall et al. (A finitevolume, incompressible Navier-Stokes model for studies of the ocean on parallel computers, submitted to Journal of Geophysical Research, 1995; Hydrostatic, quasi-hydrostatic, and nonhydrostatic ocean modeling, submitted to Journal of Geophysical Research, 1995). The model uses finite volume methods to discretize spatially and a predictor-corrector pressure correction method to step the equations forward in time.

Random noise was added to the buoyancy forcing function in order to seed the convective and baroclinic instabilities. The 
noise had half the amplitude of the mean. The density of water at temperature $\theta$ was given by $\rho=\rho_{0}\left[1-\alpha\left(\theta-\theta_{0}\right)\right]$. In experiments $H 1$ and $H 2, \theta=\theta_{0}$ initially. In experiment $S$ the initial temperature grew linearly with depth from 11.7750 to $12^{\circ} \mathrm{C}$, so that $N / f=\left[-\left(g / \rho_{0}\right) d \rho / d z\right]^{1 / 2} / f \approx 5$.

Sub-grid scale mixing was parameterized by vertical and horizontal eddy diffusivities; resolution constraints forced us to use values that produced plumes (in the reference experiments) that may be somewhat wider and more diffusive than those that exist in the real ocean. The plumes seen here are characteristic of the "heton regime" [Klinger and Marshall, 1995]. Due to its moderate resolution, our model may underestimate entrainment at the base of the mixed layer, but we expect this entrainment to be relatively small in deep convection [Anati, 1970].

For $t_{\mathrm{mix}} \approx 12$ hours and for the relatively short time step (2 min) demanded by the small grid spacing of the model, (9) implies an $\mathcal{N}_{\text {mix }}$ of less than 1 . Since the adjustment scheme requires an integer $\mathcal{N}_{\text {mix }}$, the desired value of $t_{\text {mix }}$ was attained by using $\mathcal{N}_{\text {mix }}=1$ and calling the scheme every eighth time step.

In the homogeneous experiments, for nonzero $t_{\text {mix }}$, the model tends to generate the convective plumes which were supposed to be replaced by the parameterization (when the parameterization is implemented in lower-resolution numerical experiments, the plumes will not be resolved). In order to suppress the unwanted plumes, a damping term of the form $-\lambda w$ was added to the right-hand side of the vertical momentum equation. In the unstratified experiments the damping term was applied during cooling to all points within the center of the cooling patch, using a relaxation time constant of $1 / \lambda=$ 1 hour. The outer $2 \mathrm{~km}$ of the cooling disk did not have the damping term, so that the term would not interfere with the geostrophic adjustment occuring at the edge of the cooling region. The damping term reduced the plumes to negligible amplitude and did not appear to have any extraneous effects on larger-scale flows.

Acknowledgments. Barry Klinger and John Marshall were supported by the NOAA Atlantic Climate Change Program and the Office of Naval Research. Uwe Send was supported by the European Community MAST program (contract MAST 0008-C). C. Hill offered computer assistance and B. Brown helped draft the figures. We also thank the reviewers for their many thoughtful comments.

\section{References}

Anati, D. A., On the mechanism of the deep mixed layer formation during MEDOC '69, Cah. Oceanogr., 22, 427-441, 1970.

Boubnov, B. M., and G. S. Golitsyn, Experimental study of convective structures in rotating fluids, J. Fluid Mech., 167, 503-531, 1986.

Boubnov, B. M., and G. S. Golitsyn, Temperature and velocity field regimes of convective motions in a rotating plane fluid layer, J. Fluid Mech., 219, 215-239, 1990.

Bryan, K., A numerical method for the study of the circulation of the world ocean, J. Comput. Phys., 4, 347-376, 1969.
Cox, M. A primitive equation, three-dimensional model of the ocean, GFDL Ocean Group Tech. Rep. 1, Geophys. Fluid Dyn. Lab., Princeton, N.J., 1984.

Fernando, H. J. S., R.-R. Chen, and D. L. Boyer, Effects of rotation on convective turbulence, J. Fluid Mech., 228, 513-547, 1991.

Garwood, R., S. Isakari, and P. Gallacher, Thermobaric convection, in The Polar Seas and Their Role in Shaping the Environment, Geophys. Monogr. Ser., vol. 85, edited by O. Johannessen, R. Muench, and J. Overland, pp. 199-209, AGU, Washington, D.C., 1994.

Jones, H., and J. Marshall, Convection with rotation in a neutral ocean: A study of open-ocean deep convection, J. Phys. Oceanogr., 23, 1009-1039, 1993 .

Klinger, B. A., and J. Marshall, Regimes and scaling laws for rotating deep convection in the ocean, Dyn. Atmos. Oceans, 21, 227-256, 1995.

Leaman, K. D., and F. Schott, Hydrographic structure of the convection regime in the Gulf of Lions: Winter 1987, J. Phys. Oceanogr., 21, 573-596, 1991.

Marotzke, J., Influence of convective adjustment on the stability of the thermohaline circulation, J. Phys. Oceanogr., 21, 903-907, 1991.

Marshall, J., J. A. Whitehead, and T. Yates, Laboratory and numerical experiments in oceanic convection, in Ocean Processes in Climate Dynamics: Global and Mediterranean Examples, edited by P. Malanotte-Rizzoli and A. Robinson, pp. 173-201, Kluwer Acad., Norwell, Mass., 1994.

Maxworthy, T., and S. Narimousa, Unsteady, turbulent convection into a homogeneous, rotating fluid, with oceanographic applications, $J$. Phys. Oceanogr., 24, 865-887, 1994.

MEDOC Group, Observation of formation of deep water in the Mediterranean, Nature, 227, 1037-1040, 1970.

Mertens, C., Winterliche Deckschichtentwicklung und ihre zwischenjährliche Variabilität im nordwestlichen Mittelmeer, Ph.D. diploma thesis, Univ. of Kiel, Kiel, Germany, 1994.

Paluszkiewicz, T., Greenland Sea deep convection: Results from an OGCM with a new convection parameterization, Eos Trans. $A G U$, 75(3), Ocean Sci. Meet. Suppl., 189, 1994.

Sander, J., D. Wolf-Gladrow, and D. Olbers, Numerical studies of open ocean deep convection, J. Geophys. Res., 100, 20,579-20,600, 1995.

Schott, F., and K. D. Leaman, Observations with moored accoustic Doppler current profilers in the convection regime in the Gulf of Lions, J. Phys. Oceanogr., 21, 556-572, 1991.

Schott, F., M. Visbeck, and U. Send, Open ocean deep convection, Mediterranean and Greenland Seas, in Ocean Processes in Climate Dynamics, edited by P. Malanotte-Rizzoli and A. Robinson, pp. 203-225, Kluwer Acad., Norwell, Mass., 1994.

Send, U., and J. Marshall, Integral effects of deep convection, J. Phys. Oceanogr., 25, 865-872, 1995.

Send, U., F. Schott, F. Gaillard, and Y. Desaubies, Observation of a deep convection regime with acoustic tomography, J. Geophys. Res., 100, 6927-6941, 1995.

Yin, F. L., and E. S. Sarachik, An efficient convective adjustment scheme for ocean general circulation models, J. Phys. Oceanogr., 24, 1425-1430, 1994.

B. A. Klinger, Oceanographic Center, Nova Southeastern University, Dania, FL 33004. (e-mail: klinger@ocean.nova.edu)

J. Marshall, Center for Meteorology and Physical Oceanography, Department of Earth, Atmospheric, and Planetary Sciences, Massachusetts Institute of Technology, Cambridge, MA 02139.

U. Send, Institut fuer Meereskunde, Duesternbrooker Weg 20, 24105 Kiel, Germany.

(Received November 15, 1994; revised January 9, 1996; accepted January 15, 1996.) 